ELECTROPHYSIOLOGY

\section{Radiofrequency catheter ablation of supraventricular arrhythmias}

Hugh Calkins

Johns Hopkins Hospital, Baltimore, Maryland, USA

$\mathrm{F}$ or most types of supraventricular arrhythmias medical treatment with antiarrythmic drugs is not completely effective. In addition to poor or sporadic efficacy, such drugs can be associated with a number of bothersome and even fatal side effects (although rarely), proarrhythmia, cost, and inconvenience. It is for these reasons that non-pharmacologic interventions, initially using a surgical approach and more recently utilising catheter ablation, have played an increasingly important role in the management of cardiac arrhythmias. Catheter ablation can be defined as the use of an electrode catheter to destroy small areas of myocardial tissue or conduction system, or both, that are critical to the initiation or maintenance of cardiac arrhythmias. Arrhythmias most likely to be amenable to cure with catheter ablation are those which have a focal origin or involve a narrow, anatomically defined isthmus.

Over the past two decades, catheter ablation has evolved from a highly experimental technique to first line treatment for many cardiac arrhythmias. Before 1989, catheter ablation was performed primarily with high energy direct current (DC) shocks. More recently, DC energy has been replaced with radiofrequency energy as the preferred energy source during catheter ablation procedures.

This article reviews the current state of knowledge about the technique, indications, and results of radiofrequency catheter ablation for the treatment of supraventricular cardiac arrhythmias.

\section{Technical considerations}

Catheter ablation procedures are performed in a specially equipped catheterisation laboratory, on either an inpatient or outpatient basis. Patients receive conscious sedation before and during the procedure. Two to five multipolar electrode catheters are inserted percutaneously under local anaesthesia into a femoral, brachial, subclavian, or internal jugular vein and positioned in the heart under fluoroscopic guidance. Each electrode catheter has four or more electrodes. Typically, the most distal electrode pair is used for pacing and the delivery of critically timed extra stimuli, while the proximal electrodes are used to record electrograms from localised regions within the heart. Catheter ablation is now performed primarily using radiofrequency energy. Up to $50 \mathrm{~W}$ of radiofrequency energy is delivered for $30-60$ seconds as a continuous, unmodulated, sinusoidal waveform with a frequency of approximately 500000 cycles per second, between the $4 \mathrm{~mm}$ tip of a deflectable ablation catheter and a ground plate positioned on the patient's back or chest. The majority of catheter ablation systems in use today monitor the temperature of the ablation electrode and automatically adjust power output to achieve a targeted electrode temperature of between $60-70^{\circ} \mathrm{C}$. Knowledge of the electrode temperature at a particular ablation site is useful in determining whether an unsuccessful application of radiofrequency energy failed because of inaccurate mapping or inadequate heating. ${ }^{1}$ In the event of inadequate heating, additional applications of energy at the same site with improved catheter stability may result in success. Automatic adjustment of power output using closed loop temperature control has been shown to reduce the incidence of coagulum development, which may also facilitate catheter ablation by reducing the number of times the catheter has to be withdrawn from the body to have a coagulum removed from the electrode tip.

During radiofrequency ablation, current flows into the tissue underlying the active electrode in alternating direction at high frequency. Resistive heating as a result of ionic agitation in the tissue then ensues. Thus the tissue underlying the ablation electrode, rather than the electrode itself, is the source of heat generation. The heating of tissue during radiofrequency catheter ablation may be thought of as a two step process; resistive heating followed by conductive heat transfer from the area of resistive heating to surrounding tissue. Because direct resistive heating falls precipitously with increasing distance from the ablation electrode, resistive heating is responsible for heating only a very narrow rim of tissue extending approximately $1 \mathrm{~mm}$ beyond the ablation electrode. ${ }^{1}$ The majority of lesion volume is determined by the relative contributions of conductive heat exchange into surrounding tissue and convective heat loss towards the relatively cooler moving blood.

Thermal injury is the principal mechanism of tissue destruction during radiofrequency catheter ablation procedures. Elevation of tissue temperature results in desiccation and the denaturation of proteins, and coagulation of tissue and blood. Irreversible tissue injury occurs at temperatures above $50^{\circ} \mathrm{C} .{ }^{1}$ When the temperature at the electrode tissue interface exceeds $100^{\circ} \mathrm{C}$, tissue immediately adjacent to the electrode desiccates and plasma proteins denature to form a coagulum. The development of a coagulum results in a rapid increase in impedance which leads to a dramatic decrease in current density, thereby limiting further lesion growth. As a result of the need to achieve a tissue temperature of $50^{\circ} \mathrm{C}$ for irreversible tissue injury, the $100^{\circ} \mathrm{C}$ temperature ceiling for tissue heating, and the rapid decrease in tissue temperature with increasing distance from the ablation electrode, the lesions created during radiofrequency catheter ablation procedures are small $(5 \mathrm{~mm})$ and have well demarcated borders (fig 1). Recently, 


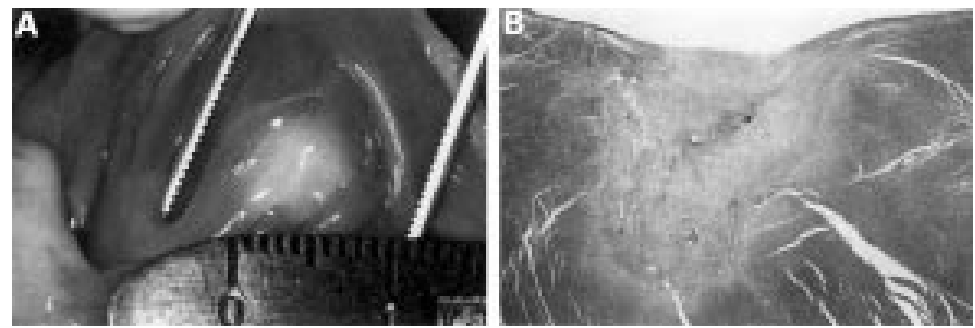

Figure 1. (A) Lesion created with radiofrequency energy one month previously in a canine ventricle. The lesion is pale, about $5 \mathrm{~mm}$ in diameter, and has smooth borders. (B) Histologic section of the same lesion. The lesion has a distinct border surrounded by normal myocardium, is hemispherical, and shows extensive fibrosis. The lesion depth is $6 \mathrm{~mm}$.

methods for improved cooling of the electrode have been developed to allow delivery of higher radiofrequency power. These include the use of larger $(8 \mathrm{~mm})$ electrodes, which receive greater convective cooling by the blood, and saline irrigated electrode tips, in which the electrode is actively cooled.

As the range of ablatable arrhythmias has broadened, the ablation procedures have, in some cases, become more technically challenging. In such cases, particularly when targeting atrial tachycardias, visualisation of the catheter tip in relation to the cardiac anatomy is crucial. Since a single fluoroscopic view displays the catheter only against the cardiac silhouette, biplane fluoroscopy is a useful addition to the electrophysiology laboratory. When the two fluoroscopic planes are placed orthogonal to each other, the position of the catheter in three dimensional space can be inferred. Several new technologies have provided a means for nonfluoroscopic tracking of catheter tip position and orientation in three dimensional space using either magnetic fields or ultrasound ranging technology. Intracardiac ultrasound is another technique which may allow better imaging of the anatomic substrate relative to the ablation catheter.

Catheter ablation of supraventricular arrhythmias

The therapeutic options for patients with supraventricular arrhythmias include pharmacologic treatment, arrhythmia surgery, and catheter ablation. The optimal management of an individual patient depends on many factors, including the type of arrhythmia, asscociated symptoms, frequency and duration of episodes, concomitant disease, and patient preference. With the exception of the Wolff-ParkinsonWhite syndrome, supraventricular arrhythmias are generally not life threatening. Thus, the inherent attractiveness of catheter ablation as a curative approach is tempered by the cost and potential complications associated with an invasive procedure. From the perspective of catheter ablation, supraventricular arrhythmias can be classified into atrial tachycardia, atrial flutter, atrial fibrillation, accessory pathways/ Wolff-Parkinson-White syndrome, and atrioventricular nodal re-entrant tachycardia (AVNRT). Catheter ablation of the atrioventricular junction is also used to control the ventricular response in some patients with supraventricular arrhythmias (mainly atrial fibrillation) which cannot be cured with catheter ablation and which are associated with a rapid ventricular response despite the use of pharmacologic treatment. The technique, results, and complications associated with ablation of atrial tachycardia, accessory pathways/ Wolff-Parkinson-White syndrome, AVNRT, and the atrioventricular junction are discussed below.

\section{Atrial tachycardia}

The term atrial tachycardia refers to a group of arrhythmias confined to the atrium which have a rate $<240$ beats per minute. Atrial tachycardias, which have a focal site of origin or result from macroreentry involving a critical isthmus of atrial tissue, are amenable to cure with radiofrequency catheter ablation. From an ablation perspective, two major types of atrial tachycardia can be considered: focal (or ectopic) atrial tachycardia, and scar mediated (or incisional) atrial tachycardia.

Focal atrial tachycardia may present as either a paroxysmal or a sustained arrhythmia. The mechanism of the tachycardia may be elucidated by pharmacologic and pacing manoeuvres, and may be classified as automatic, triggered, or re-entrant. ${ }^{2}$ The origin of these arrhythmias may be located in either the right or left atrium, usually near the pulmonary vein orifices, right atrial appendage, or crista terminalis. In the automatic and triggered tachycardias, catheter ablation is performed by manipulating one or more steerable electrode catheters in the right or left atrium to identify the site of earliest atrial activation, usually at least $30 \mathrm{~ms}$ before onset of the $\mathrm{P}$ wave. The atrial activation sequence with pacing at that site should match the activation sequence during the clinical tachycardia. In the re-entrant atrial tachycardias, appropriate ablation sites are identified using the entrainment techniques described above. Once the site is identified, $25-50 \mathrm{~W}$ of radiofrequency energy is delivered for 30-60 seconds. Results of radiofrequency catheter ablation of focal atrial tachycardias have recently been reviewed by Chen and colleagues. ${ }^{3}$ The success rate for ablation among a collective total of 252 patients included in their review was $93 \%$. The collective recurrence rate was $7 \%$.

"Inappropriate" sinus tachycardia (IST) is an ill-defined and uncommon clinical syndrome characterised by an increased resting heart rate and an exaggerated response to stress or exercise. ${ }^{4}$ The mechanism of this tachycardia is unknown, although it may involve a primary abnormality of the sinus node demonstrating enhanced automaticity or a primary autonomic disturbance with increased sympathetic activity and enhanced sinus node $\beta$ adrenergic sensitivity. The diagnosis of IST is one of exclusion. It most commonly occurs in young women, particularly those employed in the health care field. It is important to distinguish IST from the postural orthostatic tachycardia syndrome (POTS). POTS is a type of dysautonomia that typically occurs in young 
women. ${ }^{5}$ It is defined as a $>28$ beats per minute increase in heart rate on standing with associated symptoms of orthostatic intolerance such as lightheadedness and palpitations.

Patients with POTS are treated primarily with increased salt intake, fludrocortisone, and midodrine. Catheter ablation does not have a role in the management of these patients. The recognition that the sinus node region is a distributed complex exhibiting rate dependent site differentiation allows for targeted ablation to eliminate the fastest sinus rates while maintaining some degree of sinus node function. This may require some fairly extensive ablation along $3-4 \mathrm{~cm}$ of the crista terminalis. The goal may be to either modify sinus node function to prevent the fastest rates, or to ablate the sinus node completely and implant a dual chamber or atrial pacemaker. Unfortunately, only about half of affected patients receive symptomatic relief from sinus node modification. Some patients remain symptomatic even after complete sinus node ablation and pacemaker implantation, despite the absence of severe tachycardia.

There are no specific guidelines to decide which patients with IST should undergo modification of the sinus node. However, this infrequently performed procedure should be reserved for the most highly symptomatic of patients (in whom POTS has been excluded), who have failed attempts at pharmacologic treatment and are willing to undergo a procedure with considerable risk of recurrence or the need for a pacemaker, and without assurance that their symptoms will be improved.

Incisional atrial tachycardia is mediated by macroreentry around the scar of a prior surgical atriotomy. These tachycardias are frequently seen as late sequelae after surgical repair of congenital heart disease. ${ }^{6}$ Optimal ablation sites are those which occur within a protected isthmus of slow conduction that typically develops between one end of an atriotomy scar and a nearby anatomical barrier, such as the inferior or superior vena cava, or the tricuspid annulus. The extent of the atriotomy scar may be mapped by identifying sites with distinct double potentials. The two deflections are widely separated near the middle of the scar and coalesce at the ends. Entrainment techniques are used to identify optimal ablation sites within the critical isthmus. The published clinical experience with catheter ablation is small. The acute success rate has been reported to be approximately $85 \%{ }^{7}$ However, recurrences are frequent with one series reporting a recurrence rate of nearly $50 \%{ }^{7}$

Pre-excitation syndromes, Wolff-Parkinson-White syndrome, concealed pathways

Accessory pathways are anomalous extra nodal connections which connect the epicardial surface of the atrium and ventricle along the atrioventricular groove. Accessory pathways can be classified based on their location along the mitral or tricuspid annulus, type of conduction (decremental or nondecremental), and whether they are capable of antegrade conduction, retrograde conduction, or both. Accessory pathways which are capable only of retrograde conduction are concealed whereas those capable of antegrade conduction are manifest, demonstrating pre-excitation on a standard ECG. The term "Wolff-ParkinsonWhite syndrome" is reserved for patients who have both pre-excitation and symptomatic tachyarrhythmias. Among patients with the Wolff-Parkinson-White syndrome, atrioventricular reciprocating tachycardia (AVRT) is the most common arrhythmia, occurring in $75 \%$ of patients. AVRT is further subclassified into orthodromic and antidromic AVRT. During orthodromic AVRT the re-entrant impulse utilises the atrioventricular node and specialised conduction system for conduction from the atrium to the ventricle, and utilises the accessory pathway for conduction from the ventricle to the atrium. During antidromic AVRT the re-entrant impulse travels in the reverse direction with conduction from the atrium to the ventricle occurring via the accessory pathway. Atrial fibrillation is a less common but potentially more serious arrhythmia in patients with the Wolff-Parkinson-White syndrome as it can result in a very rapid ventricular response, and rarely ventricular fibrillation. The incidence of sudden cardiac death in patients with the Wolff-ParkinsonWhite syndrome has been estimated to be $0.15 \%$ per patient year.

Catheter ablation of accessory pathways is performed in conjunction with a diagnostic electrophysiology test. The purpose of the electrophysiology test is to confirm the presence of an accessory pathway, determine its conduction characteristics, and define the role of the accessory pathway in the patient's clinical arrhythmia. Accurate localisation of an accessory pathway is critical to the success of catheter ablation procedures. Among patients with pre-excitation, preliminary localisation of the accessory pathway can be determined based on the delta wave and QRS morphology. Mapping of concealed accessory pathways and more accurate localisation of manifest accessory pathways require analysis of the retrograde atrial activation sequence and/or antegrade ventricular activation sequence.

Right sided and posteroseptal accessory pathways are typically localised and ablated using a steerable electrode catheter with a $4 \mathrm{~mm}$ distal electrode positioned along the tricuspid annulus or in the coronary sinus os from the inferior vena cava. The location of left sided accessory pathways can be determined using a multipolar electrode catheter positioned in the coronary sinus, which runs parallel to the left atrioventricular groove, or with a steerable catheter positioned in the left atrium or ventricle. Once localised to a region of the heart, precise mapping and ablation is performed using a steerable $4 \mathrm{~mm}$ tipped electrode catheter positioned along the mitral annulus using either the transeptal or retrograde aortic approach. These two approaches for ablation of left sided accessory pathways are associated with a similar rate 
A

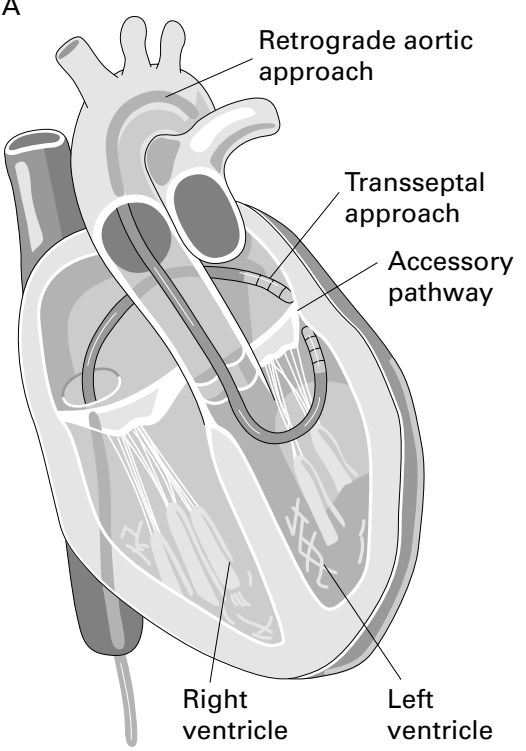

B

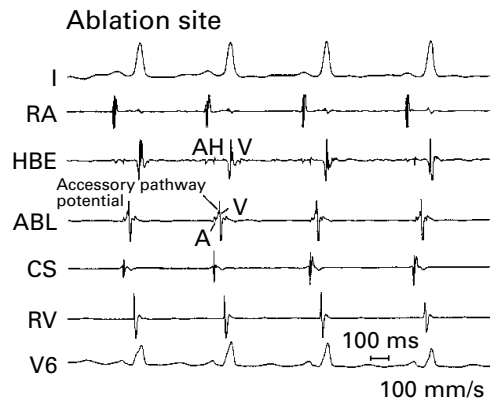

C
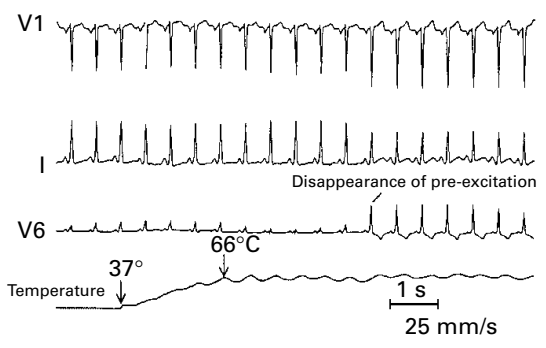

Figure 2. (A) Schematic drawing of the two approaches which are available to ablate left sided accessory pathways. The retrograde aortic approach involves inserting the ablation catheter into the femoral artery and crossing the aortic valve to enter the left ventricle. The ablation catheter is positioned against the ventricular aspect of the mitral annulus. The transseptal approach involves crossing the interatrial septum and positioning a long transeptal sheath into the left atrium. The ablation catheter is then passed through the sheath and positioned against the atrial aspect of the mitral annulus at the site of the location of the accessory pathway. (B) The electrogram characteristics of a typical successful ablation site of an accessory pathway (ABL) are shown. Also shown are the surface leads I and V6 and intracardiac recordings obtained from the high right atrium (RA), the right ventricle apex (RV), the electrode catheter positioned to record a His bundle (HBE), and an electrode catheter positioned in the coronary sinus os (CS). The surface leads show a short PR interval and slurring of the upstroke of the QRS complex, which are characteristic of the pre-excitation pattern observed in patients with the Wolff-Parkinson-White syndrome. The interval from the His bundle recording $(\mathrm{H})$ to onset of the QRS complex is less than 50 ms, confirming the presence of pre-excitation. At the successful ablation site, the ventricular electrogram (V) occurs very early relative to the onset of the QRS complex. Also observed is a discrete deflection between the atrial (A) and the ventricular components of the electrogram recorded at the ablation site, which is suggestive of an accessory pathway potential. (C) The disappearance of pre-excitation several seconds after onset of radiofrequency energy delivery during catheter ablation of an accessory pathway. Shown are the surface leads $\mathrm{V} 1$, I, V6, and the temperature recorded from the ablation catheter. The temperature recorded from the ablation electrode increases from $37^{\circ} \mathrm{C}$ to $66^{\circ} \mathrm{C}$ within two seconds of radiofrequency energy delivery. Pre-excitation resolves several seconds thereafter.

of success and incidence of complications. ${ }^{8}$ The decision over which approach to employ is usually based on physician preference, although the transeptal approach may be preferable in the elderly and in young children. In rare instances, left sided accessory pathways can only be ablated via the coronary sinus.

Appropriate sites for radiofrequency energy delivery during ablation of manifest accessory pathways are characterised by early ventricular activation, the presence of an accessory pathway potential, and stability of the local electrogram $^{9}$ (fig 2). Appropriate sites for energy delivery in patients with retrograde conduction accessory pathways mapped during ventricular pacing or orthodromic AVRT are characterised by continuous electrical activity, the presence of accessory pathway potential, and electrogram stability. Once an appropriate target site is identified, radiofrequency energy is delivered for 30-60 seconds with a target electrode temperature of $60-70^{\circ} \mathrm{C}$. At successful ablation sites, interruption of conduction through the accessory pathway usually occurs within 10 seconds, and often within two seconds of the onset of radiofrequency energy delivery (fig 2).

The reported efficacy of catheter ablation of accessory pathways varies from $89-99 \%$ with an overall success rate of approximately 93-95\%. ${ }^{10-13}$ The success rate for catheter ablation of accessory pathways is highest for left free wall accessory pathways and lowest for posteroseptal and right free wall accessory pathways (table 1). ${ }^{10-13}$ Following an initially successful procedure, recurrence of accessory pathway conduction occurs in approximately $7 \%$ of patients (fig 3). ${ }^{10-13}$ Recurrence of conduction is more common following ablation of posteroseptal and right free wall pathways. Accessory pathways which recur can usually be successfully reablated. Complications associated with catheter ablation of accessory pathways may result from obtaining vascular access (haematomas, deep venous thrombosis, perforation of the aorta, arteriovenous fistula, pneumothorax), catheter manipulation (valvar damage, microemboli, perforation of the coronary sinus or myocardial wall, coronary dissection and/or thrombosis), or delivery of radiofrequency energy (atrioventricular block, myocardial perforation, coronary artery spasm or occlusion, transient ischaemic attacks or cerebrovascular accidents). Complete heart block during ablation of an accessory pathway occurs in approximately $1 \%$ of patients, observed most commonly after ablation of septal and posteroseptal accessory pathways. The overall incidence of complications varies between $1-4 \%$. The incidence of procedure related death is estimated to be less than $0.2 \% .^{12}$

Atrioventricular nodal re-entrant tachycardia AVNRT is a common arrhythmia which occurs in patients with two functionally distinct conduction pathways through the atrioventricular node, referred to as the fast and slow pathways. The slow pathway has a shorter 
Table 1 Results of catheter ablation of the atrioventricular junction, accessory pathways, and atrioventricular nodal re-entrant tachycardia from a multicentre prospective clinical trial

\begin{tabular}{|c|c|c|c|c|c|c|}
\hline Arrhythmia & $\begin{array}{l}\text { Number of } \\
\text { patients }\end{array}$ & $\begin{array}{l}\text { Medium number (range) } \\
\text { of RF applications }\end{array}$ & $\begin{array}{l}\text { Number of patients requiring } \\
\text { second procedure for success }\end{array}$ & $\begin{array}{l}\text { Success with } \\
\text { investigational system }\end{array}$ & Overall success & Recurrence $^{\star}$ \\
\hline Atrioventricular junction & 121 & $4(1-57)$ & $3(3 \%)$ & $108(89 \%)$ & $121(100 \%)$ & $2(2 \%)$ \\
\hline AVNRT & 373 & $6(1-73)$ & $3(1 \%)$ & $348(93 \%)$ & $362(97 \%)$ & $16(5 \%)$ \\
\hline Accessory pathway & 500 & $6(1-98)$ & $24(5 \%)$ & $398(80 \%)$ & $465(93 \%)$ & $31(8 \%)$ \\
\hline LFW & 270 & $5(1-77)$ & $9(3 \%)$ & $224(82 \%)$ & $257(95 \%)$ & $7(3 \%)$ \\
\hline RFW & 92 & $9(1-98)$ & $6(7 \%)$ & $66(72 \%)$ & $83(90 \%)$ & $9(14 \%)$ \\
\hline Posteroseptal & 98 & $6(1-46)$ & $8(8 \%)$ & $73(74 \%)$ & $86(88 \%)$ & $9(12 \%)$ \\
\hline Septal & 40 & $6(1-31)$ & $1(3 \%)$ & $35(88 \%)$ & $39(98 \%)$ & $6(17 \%)$ \\
\hline Multiple accessory pathways & 36 & $16(1-54)$ & $8(22 \%)$ & $24(67 \%)$ & $31(86 \%)$ & $5(21 \%)$ \\
\hline Multiple targets & 20 & $16(2-58)$ & $4(20 \%)$ & $11(55 \%)$ & $17(85 \%)$ & $2(17 \%)$ \\
\hline Total & 1050 & $6(1-98)$ & $42(4 \%)$ & $889(85 \%)$ & $996(95 \%)$ & $56(6 \%)$ \\
\hline
\end{tabular}

^Analysis of arrhythmia recurrence was confined to those patients in whom success was achieved with the investigational ablation system.

AVNRT, atrioventricular nodal re-entrant tachycardia; LFW, left free wall; RFW, right free wall; RF, radiofrequency.

Reproduced from Calkins et al, Circulation 1999;99:262-70, with permission of the publisher.

refractory period than the fast pathway. Both the fast and slow pathways are necessary to maintain AVNRT. The common form of AVNRT is typically initiated when an atrial premature beat blocks the fast pathway, conducts down the slow pathway, and returns via the fast pathway to depolarise the atrium. During the uncommon form of AVNRT the wave front propagates in the opposite direction, conducting down the fast pathway and returning via the slow pathway. The fast pathway is located anteriorly along the septal

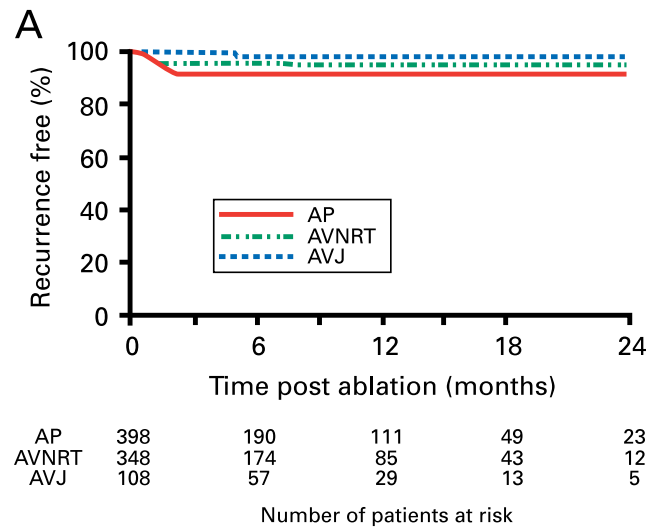

B

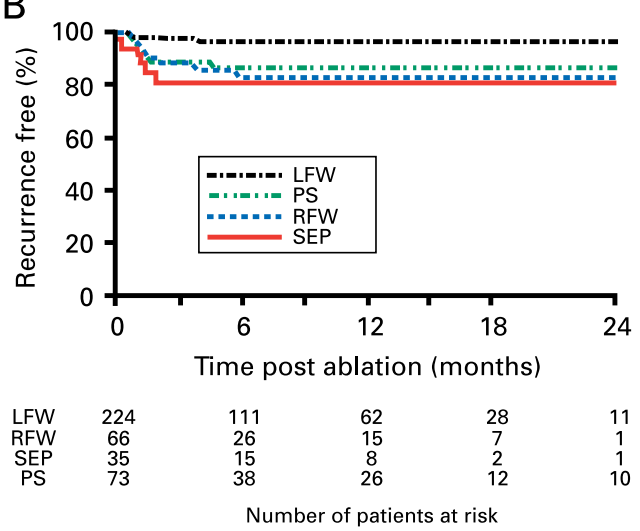

Figure 3. (A) Kaplan-Meier curve showing freedom from arrhythmia recurrence among patients who underwent successful ablation of an accessory pathway (AP), atrioventricular nodal re-entrant tachycardia (AVNRT), or atrioventricular junction (AVJ). (B) Kaplan-Meier curve showing freedom from arrhythmia recurrence among patients who underwent successful ablation of an accessory pathway subclassified by its location. LFW, left free wall; RFW, right free wall; PS, posteroseptal; SEP, septal. Reproduced from Calkins et al, Circulation 1999;99:262-70, with permission of the publisher. portion of the tricuspid annulus, near the compact atrioventricular node, whereas the atrial insertion of the slow pathway is located more posteriorly along the tricuspid annulus, closer to the coronary sinus os.

AVNRT may be cured by ablation of either the fast or the slow pathway. These alternative approaches are referred to as the "anterior" and the "posterior" approaches respectively. The anterior approach targets the fast pathway. Catheter ablation is performed by locating an electrogram with a large His potential and then withdrawing the ablation catheter into the right atrium until the atrial signal is at least twice that of the ventricular signal $(\mathrm{A}: \mathrm{V}$ ratio $>2)$ with a His potential no larger than $50 \mu \mathrm{V}$. Radiofrequency energy is then applied during sinus rhythm for 30-60 seconds while watching for prolongation in the PR interval. Energy delivery is immediately terminated if atrioventricular block occurs.

Successful ablation of AVNRT using the anterior approach is characterised by lengthening of the PR interval and the inability to induce the tachycardia. Typically, there is 2 elimination or pronounced attenuation of retrograde conduction during ventricular pacing. The atrioventricular block cycle length and the atrioventricular node effective refractory period are not usually altered during ablation of AVNRT using the anterior approach. Catheter ablation of AVNRT using the anterior approach is successful in approximately $90 \%$ of patients. Major limitations of the technique are the creation of inadvertent atrioventricular block in approximately $7 \%$ of patients and a $9 \%$ incidence of recurrence.

The posterior approach to ablation of AVNRT targets the slow pathway. The ablation catheter is directed into the right ventricle low near the posterior septum and is then withdrawn until an electrogram is recorded with a small atrial electrogram and a large ventricular electrogram (A:V ratio < 0.5$)$. Specific ablation sites along the posterior portion of the tricuspid annulus can be selected based either on the appearance of the local atrial electrogram or based strictly on anatomic factors. When using the electrogram guided approach, fractionated atrial electrograms with a late "slow potential" are targeted (fig 4). When using the anatomic approach, the initial applications are delivered at the level of the coronary 


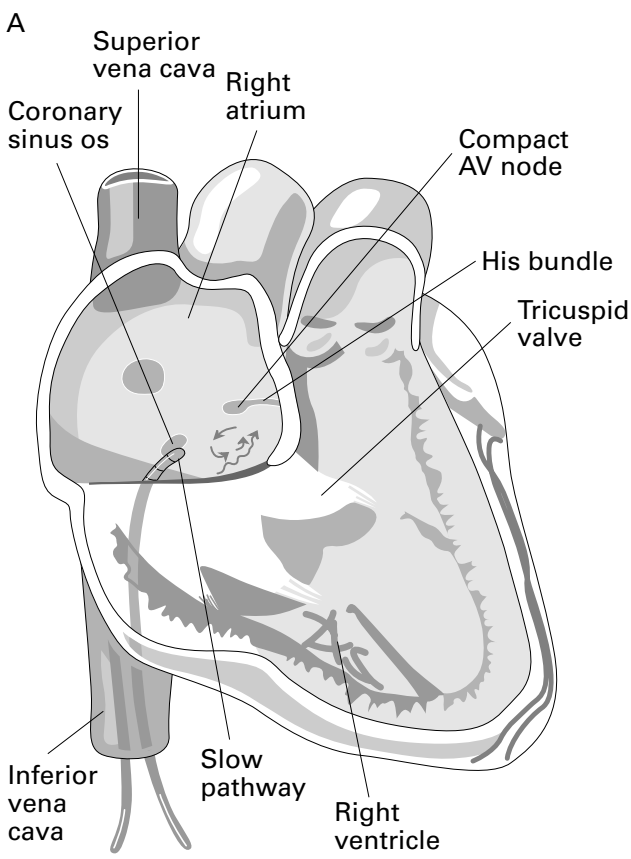

B

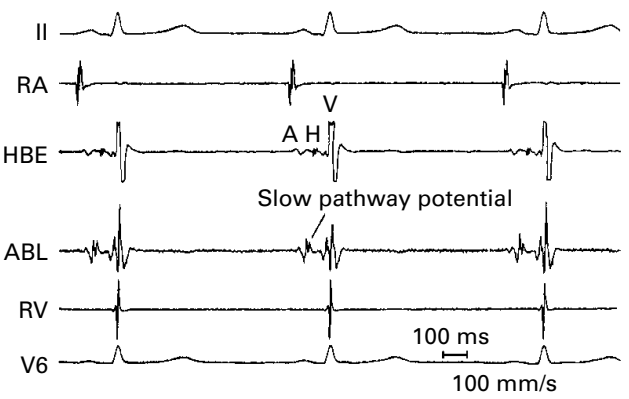

Figure 4. (A) Schematic drawing of the posterior or "slow pathway" approach to catheter ablation of atrioventricular nodal re-entrant tachycardia (AVNRT). The ablation catheter is positioned anterior to the coronary sinus os. A schematic drawing of the hypothesised re-entrant circuit for AVNRT is also shown. (B) The electrogram of a successful slow pathway ablation site. Also shown are surface leads 1 and V 6 and intracardiac recordings obtained from the right atrium, the right ventricle, and the electrode catheter positioned to record a His bundle. The

intracardiac electrogram at the ablation site reveals a ventricular electrogram substantially larger than the atrial electrogram, and a distinct deflection consistent with a slow pathway potential immediately after the atrial electrogram. The arrow points to a slow pathway potential.

sinus os with subsequent applications of energy delivered to more superior sites.

With either approach, junctional beats occurring during the application of radiofrequency energy are a marker for successful ablation. Successful ablation of AVNRT using the posterior approach is characterised by an increase in the atrioventricular block cycle length and in the atrioventricular node effective refractory period and elimination of inducible AVNRT. The posterior approach for ablation of AVNRT is effective in greater than $95-97 \%$ of patients (table 1). ${ }^{12} 1415$

Atrioventricular block is the most common complication, occurring in $0.5-1 \%$ of patients.
The incidence of recurrence following successful ablation of AVNRT using the posterior approach is approximately 3\% (fig 3). Because of the higher efficacy, lower incidence of atrioventricular block and arrhythmia recurrence, and the greater likelihood of maintaining a normal PR interval during sinus rhythm, the posterior approach is now considered the preferred approach to ablation of AVNRT.

Atrioventricular junction

Theoretically, catheter ablation of the atrioventricular junction can eliminate any type of supraventricular arrhythmia which utilises the atrioventricular node as part of the re-entrant circuit, and can slow the ventricular response to supraventricular arrhythmias confined to the atrium. In practice, catheter ablation of the atrioventricular junction is reserved for atrial arrhythmias which cannot be controlled with pharmacologic treatment and which result in a rapid ventricular response.

The procedure is performed by positioning a steerable ablation catheter across the tricuspid annulus to record the largest $\mathrm{His}$ bundle electrogram associated with the largest atrial electrogram. A second electrode catheter is placed at the apex of the right ventricle for temporary pacing. Once an appropriate target site is identified, radiofrequency energy is delivered for 30-60 seconds. If atrioventricular conduction remains unchanged, the catheter is repositioned and a repeat attempt is made. If unsuccessful, a left sided approach can be used. ${ }^{16}$ The ablation catheter is passed retrogradely across the aortic valve into the left ventricle and positioned immediately below the aortic valve to record a His bundle electrogram. Radiofrequency energy is then delivered in a standard fashion. The overall efficacy of catheter ablation of the atrioventricular junction using these two approaches is $100 \%$ (table 1). ${ }^{12} 16-18$

Following ablation of the atrioventricular junction a permanent rate responsive pacemaker is inserted. Complication rates are generally less than $2 \%$ with an estimated incidence of procedure related death of $0.2 \% .{ }^{12}$ Late sudden death has been reported following DC or radiofrequency ablation of the atrioventricular junction. Because many of these patients have severe underlying heart disease, it is difficult to attribute these late sudden deaths directly to the ablation procedure.

\section{Economic considerations}

The cost of catheter ablation procedures compares favourably with that of arrhythmia surgery or lifelong antiarrhythmic treatment. In one US study, the cost of surgery in patients with Wolff-Parkinson-White syndrome was estimated to be $\$ 53000$, compared with $\$ 15000$ for catheter ablation. ${ }^{19}$ Another study, which compared the cost of medical management before catheter ablation with the cost of the procedure in patients with AVNRT, found total costs of $\$ 7600$ and $\$ 16000$, respectively. ${ }^{20}$ The investigators estimated that the 


\section{Key points}

- Catheter ablation is now considered as a first line alternative to pharmacologic therapy for the treatment of focal atrial tachycardia, atrioventricular nodal re-entrant tachycardia, and atrioventricular reciprocating tachycardia associated with an accessory pathway

- Catheter ablation of the atrioventricular junction is also commonly used to control the ventricular response in patients with atrial fibrillation

Arrhythmias recur following 3-5\% of successful catheter ablation procedures

- Recurrences generally present within the first three months following an ablation procedure

A repeat ablation procedure is associated with a very high likelihood of long term success

annual medical costs, including medications and an annual office visit, for a patient whose tachycardia is effectively controlled with verapamil, for example, would equal the cost of catheter ablation in approximately 15 years. These cost effective ratios are within the range generally thought to warrant adoption of the procedures. Thus, from an economic perspective, the expense of catheter ablation appears justifiable.

\section{Conclusion}

The safety and efficacy of radiofrequency catheter ablation for treatment of most types of supraventricular arrhythmias is well established. These arrhythmias/arrhythmia substrates include AVNRT, accessory pathways, and focal atrial tachycardia. Because of this catheter ablation is considered as an alternative to pharmacologic therapy in the treatment of these cardiac arrhythmias. The technique, safety, and efficacy of catheter ablation for treatment of atrial fibrillation remains an area of active research. Although the potential for catheter ablation of atrial fibrillation has been demonstrated, further research is needed to approach the remarkably high safety, efficacy, and ultimately clinical acceptance which has been seen with catheter ablation of most other types of supraventricular arrhythmias.

1. Dinerman J, Berger RD, Calkins H. Temperature monitoring during radiofrequency ablation. $J$ Cardiovasc Electrophysiol 1996;7:163-73.

- This is a recent and comprehensive review of the literature concerning the biophysics of radiofrequency catheter ablation and the clinical role of temperature monitoring.

2. Chen SA, Chiang CE, Yang CJ, et al. Sustained atrial tachycardia in adult patients. Electrophysiological characteristics, pharmacological response, possible mechanisms, and effects of radiofrequency ablation. Circulation 1994;90:1262-78.

- A description is provided of the technique, results, and complications associated with catheter ablation of atrial tachycardias.
3. Chen SA, Tai CT, Chiang CE, et al. Focal atrial tachycardia: reanalysis of the clinical and electrophysiologic characteristics and prediction of successful radiofrequency ablation. J Cardiovasc Electrophysiol 1998;9:355-65.

4. Lee, RJ, Kalman JM, Fitzpatrick AP, et al. Radiofrequency catheter modification of the sinus node for "inappropriate" sinus tachycardia. Circulation

1995;92:2919-28.

5. Low PA, Opfer-Gehrking TL, Textor SC, et al. Postural tachycardia syndrome (POTS). Neurology 1995;45(suppl 5):S19-25.

6. Lesh MD, Kalman JM, Saxon LA, et al.

Electrophysiology of "incisional" reentrant atrial tachycardia complicating surgery for congenital heart disease. PACE 1997;20:2107-11.

7. Baker BM, Lindsay BD, Bromberg BI, et al. Catheter ablation of clinical intraatrial reentrant tachycardias resulting from previous atrial surgery: localizing and transecting the critical isthmus. J Am Coll Cardiol 1996;28:411-17.

8. Lesh MD, Van Hare G, Scheinman MM, et al. Comparison of the retrograde and transseptal methods for Cardiol 1993;22:542-9.

- This article describes, and prospectively compares, the retrograde aortic and the transeptal approach to catheter ablation of left sided accessory pathways.

9. Calkins H, Kim YN, Schmaltz S, et al. Electrogram criteria for identification of appropriate target sites for radiofrequency catheter ablation of accessory atrioventricular connections. Circulation 1992;85:565-73.

10. Jackman WM, Wang $\mathbf{X}$, Friday KJ, et al. Catheter ablation of accessory atrioventricular pathways

(Wolff-Parkinson-White syndrome) by radiofrequency current. N Engl J Med 1991:324:1605-11.

- This landmark article describes the technique and results of radiofrequency catheter ablation of accessory pathways.

11. Calkins H, Sousa J, El-Atassi R, et al. Diagnosis and cure of the Wolff-Parkinson-White syndrome or paroxysma supraventricular tachycardias during a single

electrophysiology test. N Engl J Med 1991;324:1612-18.

- A further landmark article describing the technique and results of radiofrequency catheter ablation of results of radionrequency catheter ablation of atrioventricular nodal re-entrant tachycardia and accessory pathways. This study was also the first to dem electrophysiology test and catheter ablation in a single setting.

12. Calkins H, Yong P, Miller JM, et al, for the Atakr Multicenter Investigators Group. Catheter ablation of accessory pathways, atrioventricular nodal reentran tachycardia, and the atrioventricular junction: final results of a prospective, multicenter clinical trial. Circulation 1999;99:262-70.

- This describes the results of the only prospective multicentre clinical trial of radiofrequency catheter ablation of accessory pathways, atrioventricular nodal re-entrant tachycardia, and the atrioventricular junction.

13. Calkins H, Prystowsky E, Berger RD, et al, and the Atakr Multicenter Investigators Group. Recurrence of conduction following radiofrequency catheter ablation procedures: relationship to ablation target and electrode procedures: relationship to ablation target and electrode

14. Haissaguerre M, Gaita F, Fischer B, et al. Elimination of atrioventricular nodal reentrant tachycardia using discrete slow potentials to guide application of radiofrequency energy. Circulation 1992;85:2162-75.

15. Jackman WM, Beckman KJ, McClelland JH, et al. Treatment of supraventricular tachycardia due to atrioventricular nodal reentry by radiofrequency catheter ablation of slow-pathway conduction. $N$ Engl J Med 1992;327:313-18.

- This is the first article to report the technique, results, and complications associated with catheter ablation of atrioventricular nodal re-entrant tachycardia using the posterior or "slow pathway" approach. This approach is now considered the standard approach.

16. Sousa J, El-Atassi R, Rosenheck S, et al. Radiofrequency catheter ablation of the atrioventricula junction from the left ventricle. Circulation 1991;84:567-71.

17. Curtis AB, Kutalek SP, Prior M, et al. Prevalence and characteristics of escape rhythms after radiofrequency ablation of the atrioventricular junction: results from the registry for atrioventricular junction ablation and pacing in atrial fibrillation. Am Heart J 2000;139:122-5.

18. Scheinman MM, Huang S. The 1998 NASPE prospective catheter ablation registry. Pacing Clin Electrophysiol 2000;23:1020-8.

19. De Buitleir M, Sousa J, Bolling SF, et al. Reduction in medical care cost associated with radiofrequency catheter ablation of accessory pathways. Am J Cardiol 1991;68:1656-61.

20. Kalbfleisch SJ, El-Atassi R, Calkins H, et al. Safety, feasibility and cost of outpatient radiofrequency catheter ablation of accessory atrioventricular connections. J Am Coll Cardiol 1993;21:567-70. 